\title{
Modeling \& simulation of Missile Fire Allocation of Warship Formation Based on Improved Genetic Algorithm
}

\author{
Feng Jie \\ Dalian Navel Academy, Dalian 116018, China \\ Fdf8821@163.com
}

\begin{abstract}
.
Aiming at the missile fire allocation of warship formation, a valuation method of damage target value coefficient is put forward. According to the amount of carried anti-warship missile by attacking warships, the model of missile fire allocation is built up. Two methods of improved genetic algorithm are put forward, which are two class code method and the invariable biggest number of the control condition, and the running speed of the algorithm is enhanced. Simulation calculation is carried out by use of the algorithm to the model, and the project of missile fire allocation is found which is kept with battle demand and provided support for military commanders' assistance decision.
\end{abstract}

Keyword: : Modeling \& simulation, Improved genetic algorithm, Two class code, Value coefficient, Missile fire allocation

\section{Introduction}

Warship formation is a main constitute part of the navy surface warship, which is also important sudden attack strength of sea stroke enemy warship. According to the battle mission, one guided missile warship, group or formation can attack to an enemy surface warship by missiles. How according to superior's intention, battle mission and carried anti-warship guided missile amount, reasonably allocate guided missile amount to concrete enemy formation circumstance, and obtain the best results in battle, which is first problem to be solved of commander when command anti-warship battle. When guided missiles attack to many surface warships, the optimal fire allocation problem is always face of one difficulty topic of the military tactics [1-2]. Reference [3] researches improved genetic algorithm application to ground-ground tactics missile fire allotment, Reference [4] researches improved genetic algorithm application to guided missile. Aiming at the missile fire allocation of warship formation, a valuation method of damage target value coefficient is put forward. The model of missile fire allocation is built up. Two methods of improved genetic algorithm are put forward. Simulation calculation is carried out by use of the algorithm to the model, and the project of missile fire allocation is found which is kept with battle demand. 


\section{Modeling of Missile Fire Allocation}

\subsection{The damage probability of one missile to one target}

The damage probability $W$ of one missile to one target is equal to the product of $P$ and damage probability: $W=P \cdot G$. The damage probability of one target when lunched $m$ missiles to $n$ targets is equal to the sum of damage probability of missiles to the target: $W_{m}=\sum_{i=1}^{m} P_{i, n} G(i)$

\subsection{The damage probability of missiles to the formation}

When the red anti-warship missiles shoot to $n$ targets in the enemy formation simultaneously, first The damage probability of missiles to enemy targets is calculated individually, then According to the values, the total damage probability of enemy warship formation is computed integrality. Supposed that there are $m$ types missile whose sequential number is $i$, whose total missile amount is $m_{i}$, the total amount of enemy targets is $n$, whose sequential number is $j$, and the damage probability of the $i$ th type missile to the $j$ th target is $r_{i j}$. For some formation, supposed that the missile fire allocation plan is already assured, whose missile fire allocation matrix is as follows:

$$
X=\left(x_{i j}\right)_{m \times n}=\left[\begin{array}{cccc}
x_{11} & x_{12} & \ldots & x_{1 n} \\
x_{21} & x_{22} & \ldots & x_{2 n} \\
\ldots & \ldots & \ldots & \ldots \\
x_{m 1} & x_{m 2} & \ldots & x_{m n}
\end{array}\right]
$$

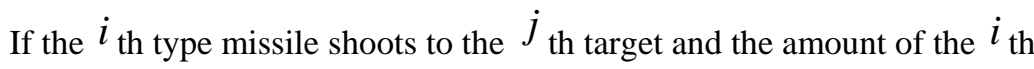
type missile is $X_{i j}$, then the damage probability of one target is as follows:

$$
w_{i j}=1-\left(1-r_{i j}\right)^{x_{i j}}
$$

The damage probability of $m_{\text {types to target }} j$ is as follows:

$$
W_{j}=1-\prod_{i=1}^{m}\left(1-r_{i j}\right)^{x_{i j}}
$$




\subsection{Modeling of missile fire allocation}

The object function of the optimal missile fire allocation makes the damage value of whole warship formation optimize, i.e. makes the expectation of the weighted damaging targets optimize. Supposed that the value coefficient vector is $K=\left(k_{1}, k_{2}, \cdots, k_{i}, \cdots, k_{n}\right)$, in which $k_{i}$ is represented as the value coefficient of $i$ th target, whose computational method is according to optimization principal of strategy tactics value and the following factors are considered: (1) Degree according to battle mission $S M=\left(s m_{1}, s m_{2}, \ldots, s m_{n}\right) ;(2)$ Value coefficient of enemy target $J Z=\left(j z_{1}, j z_{2}, \ldots, j z_{n}\right)$; (3) Threat degree of enemy target $W X=\left(w x_{1}, w X_{2}, \ldots, w X_{n}\right) \quad ;(4)$ frigidly attack of enemy target $Y G=\left(j g_{1}, j g_{2}, \ldots, j g_{n}\right)$. The above principals are integrated and the strategy tactics value vector is obtained: $K=\left(k_{1}, k_{2}, \cdots, k_{i}, \cdots, k_{n}\right)=(S M \otimes W X \otimes J Z \otimes J G \otimes)$. Therefore, the model of the optimal missile fire allocation is as follows:

$$
\begin{aligned}
& \max W=\sum_{j=1}^{n} k_{j}\left[1-\prod_{i=1}^{m}\left(1-r_{i j}\right)^{x_{i j}}\right] \\
& \text { s.t. }\left\{\begin{array}{l}
\sum_{j=1}^{n} x_{i j} \leq m_{i}, \\
x_{i j} \geq 0, \\
i=1,2, \ldots \ldots, m, \\
j=1,2, \ldots \ldots, n .
\end{array}\right.
\end{aligned}
$$

This is a nonlinear integral programming problem, which can be solved by traditional number method. But the traditional calculation method may have great work capacity, and sometimes is not easy to get the optimal solution. We try to use improved genetic algorithm to solve this problem.

\section{Improved Genetic Algorithm}

\subsection{Two class coding method}

The basic principle of two class coding method: First, an entry-level coding of problem is carried on, namely the variable is sign coded and its quantity scope is limited; then, according to sign of variable limit, the second class is coded. The fire allocation matrix $X$ is coded according to ${ }^{X_{i j}}$ regard as a variable. First class coding: $\left(x_{11} \cdots x_{1 n} x_{21} \cdots x_{2 n} \cdots \cdots x_{m 1} \cdots x_{m n}\right), 0 \leq x_{i j} \leq 8$;Second 
class coding: $\left(b_{1} b_{2} b_{3} \cdots b_{(3 \times m \times n)-2} b_{(3 \times m \times n)-1} b_{(3 \times m \times n)}\right)$. In which coded length $l$ is : $l=3 \times m \times n ;$ Code zone is: $U_{\min }=0, \quad U_{\max }=2^{3 \times m \times n}$, $\left[0,2^{l}\right]=\left[0,2^{3 \times m \times n}\right]$; Code accuracy is: $\delta=\frac{U_{\max }-U_{\min }}{2^{3 \times m \times n}-1}$; and decoded formula is:

$$
x=U_{\min }+\left(\sum_{i=1}^{l} b_{i} \cdot 2^{i-1}\right) \cdot \frac{U_{\max }-U_{\min }}{2^{l}-1} .
$$

\subsection{The method of invariable biggest number of limit condition}

The thought of this method is: According to the quantity limit of the variable in particular problem, the up boundary $U_{\max }$ and down boundary $U_{\text {min }}$ are assured. When genotic operation is carried on, if one among those performance type of decoding value is $x_{i j}>U_{\max }$ or $x_{i j}<U_{\min }$, the method of invariable biggest number of limit condition is adopted. When $x_{i j}>U_{\max }$, calculating the quantity outrun up the boundary of the variable, then minus the quantity of the variable which is related with them. under the most circumstances new performance type of decoding value matches scope limit.

\subsection{Individual fitness}

Red consume minimum principle: supposed that the missile value vector of red warship formation and the missile consumed vector is $F=\left(f_{1}, f_{2}, \cdots, f_{m}\right)$, and then the optimal object function of red's consumed value is: $C=\left(c_{1}, c_{2}, \cdots, c_{m}\right)$, then the optimal object function of red's consumed value is:

$$
\begin{aligned}
& \min Z=F * C \\
& \text { s.t. } \quad\left\{\begin{array}{l}
0 \leq f_{i} \leq f_{\max }, \\
0 \leq c_{i} \leq C_{\max }, \\
i=1,2, \cdots, m .
\end{array}\right.
\end{aligned}
$$

According to principles of the biggest value (4) and minimum consumed (5), comprehensive evaluation function is obtained:

$$
W_{\text {总 }}=W-\alpha Z=\sum_{j=1}^{n} k_{j}\left[1-\prod_{i=1}^{m}\left(1-r_{i j}\right)^{a_{i j}}\right]-\alpha \cdot \sum_{i=1}^{m} f_{i} \cdot c_{i}
$$

In which $\alpha$ is revising coefficient and balances quantity and in general it is a expirenced number. 
The propability of Individual heredity to next generation is assured by individual fitness. for the maximumu problem, the fitness function is adopted as follows:

$$
f(x)=\left\{\begin{array}{cc}
g(x)-c_{\min } & \text { if } g(x)-c_{\min }>0 \\
0 & \text { otherwise }
\end{array}\right.
$$

In which $C_{\text {min }}$ is the minimum value of $g(x)$ in current all generations or recent generations $K, g(x)$ is object function value in current generation individual.

\subsection{Genetic operator design}

Selection operator: fitness proportional selection method is adopted. For given population $n$, the fitness of $a_{i}$ is $f\left(a_{i}\right)$, whose selection propability is $p_{s}\left(a_{j}\right)=f\left(a_{j}\right) / \sum_{i=1}^{n} f\left(a_{i}\right),(j=1,2, \cdots, n)$

Crossover operator: unversal crossover method is used. For example, $s_{1}^{\prime}=a_{11}^{\prime} a_{12}^{\prime} \cdots a_{1 L}^{\prime}$ $S_{2}^{\prime}=a_{21}^{\prime} a_{22}^{\prime} \cdots a_{2 L}^{\prime}, O\left(p_{c}, x\right): a_{1 i}^{\prime}=\left\{\begin{array}{ll}a_{1 i}, & x>1 / 2 \\ a_{2 i}, & x \leq 1 / 2\end{array}, a_{2 i}^{\prime}=\left\{\begin{array}{ll}a_{2 i}, & x>1 / 2 \\ a_{1 i}, & x \leq 1 / 2\end{array}\right.\right.$.

Mutation operator: When mutation operation is carried out from $s=a_{1} a_{2} \cdots a_{k} \cdots a_{L}$ to $s^{\prime}=a_{1} a_{2} \cdots a_{k}^{\prime} \cdots a_{L}$, if genetic value scope is $\left[U_{\min }^{k}, U_{\max }^{k}\right]$ at mutation point $a_{k}$ and supposed that $\mu=\left(U_{\max }^{k}+U_{\min }^{k}\right) / 2, \quad \sigma=\left(U_{\max }^{k}-U_{\min }^{k}\right) / 6$, then new genetic value $x_{k}^{\prime}$ is assured by $x_{k}^{\prime}=\left(U_{\max }^{k}+U_{\min }^{k}\right) / 2+\left(U_{\max }^{k}-U_{\min }^{k}\right) / 6 \bullet\left(\sum_{i=1}^{12} r_{i}-6\right)$.

\subsection{Run parameters set}

Chromosome length $l$ : supposed that $\boldsymbol{m}$ is red warships, $\boldsymbol{n}$ is blue warships, then $l=3 \times m \times n$. Population size $\boldsymbol{M}=20 \sim 30$. Crossover probability $\boldsymbol{P}_{c}=0.3 \sim 0.65$. Mutation probability $\boldsymbol{P}_{m=0.001 \sim 0.005}$. Terminal generations $T=800 \sim 1200$. Initial population: the probability of generation of " 1 " is 0.6 , the probability of generation of " 0 ” is 0.4 .

\section{Simulation calculation}


The parameters of missile fire allocation of warship formation is set as follows: red warships: 5 , blue warships: 6 , the same type of missile vector which red warship formation carried is $(8,16,8,8,8)$, when attack effect of red warship formation missiles is damage, whose damage probability matrix is:

$$
R=\left(r_{i j}\right)_{5 \times 6}=\left[\begin{array}{cccccc}
0.42 & 0.54 & 0.39 & 0.39 & 0.39 & 0.39 \\
0.25 & 0.36 & 0.24 & 0.24 & 0.24 & 0.24 \\
0.25 & 0.36 & 0.24 & 0.24 & 0.24 & 0.24 \\
0.25 & 0.36 & 0.24 & 0.24 & 0.24 & 0.24 \\
0.25 & 0.36 & 0.24 & 0.24 & 0.24 & 0.24
\end{array}\right]
$$

\section{Supposed}

$\mathrm{JZ}=(0.35,0.18,0.18,0.18,0.18)$

$J Z=(0.25,0.15,0.1,0.1,0.2,0.2)$

$W X=(0.3,0.1,0.1,0.1,0.18,0.22), Y G=(0.5,1.0,1.0,1.0,0.6,0.6)$ that

$$
S M=(1.0,0.7,0.8,0.8,1.0,1.0)
$$

Run parameters of improved genetic algorithm is set as follows: $T=1000$; M $=20, \boldsymbol{P}_{c}=0.55, \boldsymbol{P}_{m}=0.003$. MATLAB 7.3 is used as a simulation tool. As shown in fig.1, the calculating output of individual chromosome fitness of preceding 50 generation is plotted. Seen from which the chromosome fitness distribution of preceding 10 generation is disseminated broadly, this is because chromosome bit strings are generated by random. As shown in fig.2, the calculating output of individual chromosome fitness of 20 generation from 1 to 1000. Seen from which the chromosome fitness is converged to the optimal value 3.331410, which is biggest value we need: “011001000000000000000000000000110001000000001101000000000011100 000000000000000000000000101 ", the missile fire allocation matrix is decoded as follows.

$$
X=\left(x_{i j}\right)_{5 \times 6}=\left[\begin{array}{l}
3,1,0,0,0,0 \\
0,0,0,0,6,1 \\
0,0,0,6,0,0 \\
0,3,4,0,0,0 \\
0,0,0,0,0,5
\end{array}\right]
$$



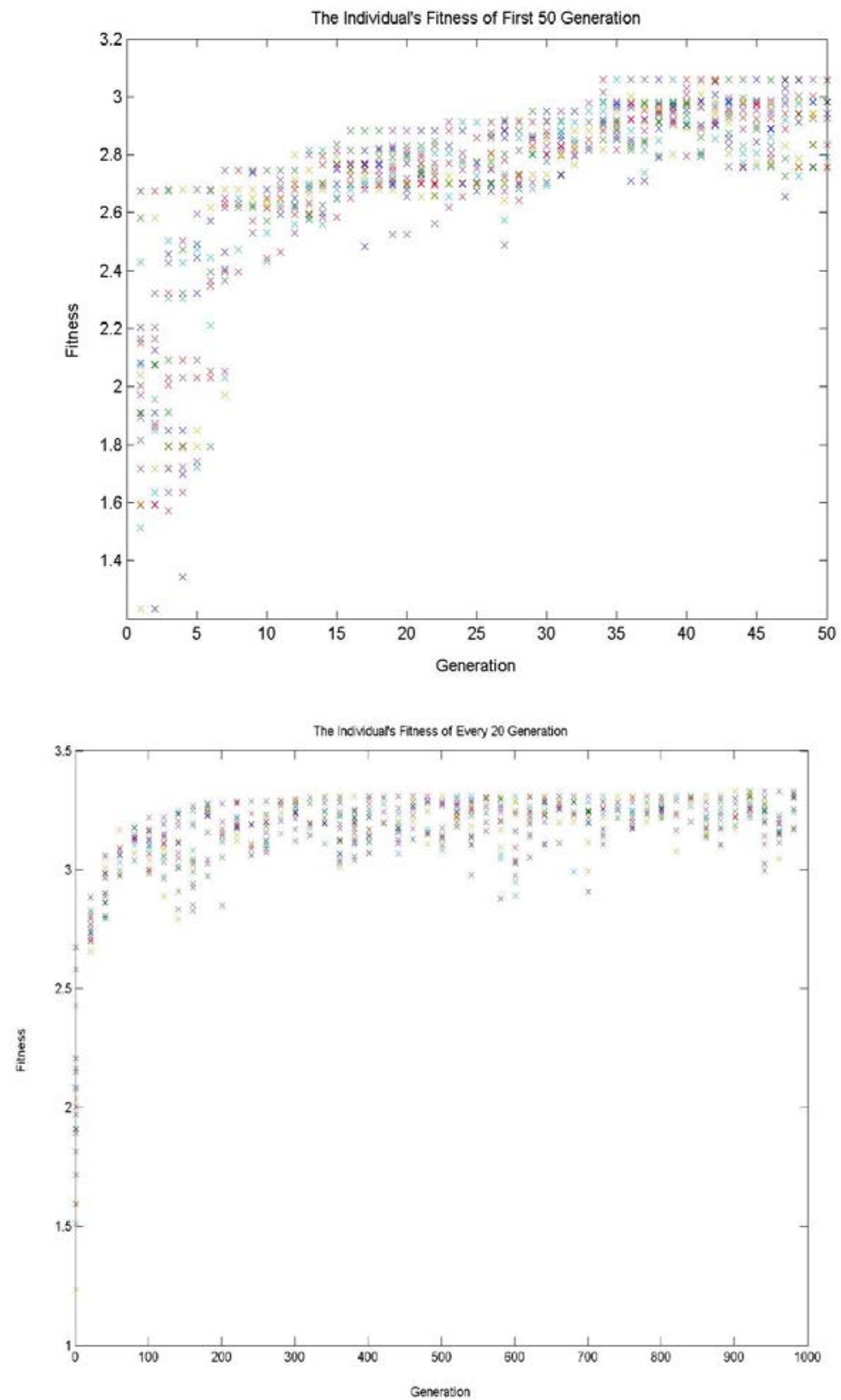

Fig.1 The fitness preceding 50 generations Fig.2 the fitness of 20 generations from 1 to 1000 
Let $X$ into (3), missile attack effect of warship formation is $W=(0.815,0.733,0.833,0.925,0.925,0,925)$ which can be meted with the tactics requirement.

\section{Summary}

Aiming at the missile fire allocation of warship formation, a valuation method of damage target value coefficient is put forward. Considering the amount of carried anti-warship missile by attacking warships, the model of missile fire allocation is built up. Two methods of improved genetic algorithm are put forward, which are two class code method and the invariable biggest number of the control condition, and the running speed of the algorithm is enhanced. Simulation calculation is carried out by use of the algorithm to the model, and the project of missile fire allocation is found which is kept with battle demand and provided support for military commanders' support decision.

\section{References}

[1] Zhang yong sheng etc.: Researech on modeling and application of anti-warship missile fire allocation [J], warship electronics engineering, $(2009,11)$

[2] Feng Jie: Genetic algorithm and its application in missile fire allocation [J], Fire and command control, $(2004,12)$

[3] He Xinfeng etc.: Improved genetic algorithm and its application in missile fire allocation, Fire and command control [J], $(2007,3)$

[4] Wang Guangyuan etc.: Research on missile fire allocation based on improved genetic algorithm [J]. Solider automation, (2012, 31(5)), P.5-8

[5] Chen Jianhua etc.: Warship-air missile fire optimal simulation based on improved genetic algorithm [J], Computer simulation (2010, 2) P.1-9 